\title{
Multi-profile intertemporal social choice: a survey*
}

\author{
WALter Bossert \\ Department of Economics and CIREQ \\ University of Montreal \\ P.O. Box 6128, Station Downtown \\ Montreal QC H3C 3J7 \\ Canada \\ FAX: (+1 514) 3437221 \\ e-mail: walter.bossert@umontreal.ca \\ and \\ Kotaro Suzumura \\ School of Political Science and Economics \\ Waseda University \\ 1-6-1 Nishi-Waseda \\ Shinjuku-ku, Tokyo 169-8050 \\ Japan \\ FAX: (+81 3) 52861818 \\ e-mail: ktr.suzumura@gmail.com
}

This version: January 28, 2013

* This paper is dedicated to Nick Baigent in appreciation of his invaluable contribution to the academic community. We thank two referees for their thoughtful comments and suggestions for improvements. Financial support from a Grant-in-Aid for Specially Promoted Research from the Ministry of Education, Culture, Sports, Science and Technology of Japan for the Project on Economic Analysis of Intergenerational Issues (grant number 22000001), the Fonds de Recherche sur la Société et la Culture of Québec and the Social Sciences and Humanities Research Council of Canada is gratefully acknowledged. 


\begin{abstract}
We provide a brief survey of some literature on intertemporal social choice theory in a multi-profile setting. As is well-known, Arrow's impossibility result hinges on the assumption that the population is finite. For infinite populations, there exist nondictatorial social welfare functions satisfying Arrow's axioms and they can be described by their corresponding collections of decisive coalitions. We review contributions that explore whether this possibility in the infinite-population context allows for a richer class of social welfare functions in an intergenerational model. Different notions of stationarity formulated for individual and for social preferences are examined. Journal of Economic Literature Classification No.: D71.
\end{abstract}

Keywords: Infinite-population social choice, multi-profile social choice, decisiveness, intergenerational choice. 


\section{Introduction}

The conclusion of Arrow's (1951; 1963; 2012) dictatorship theorem depends on the assumption that the population under consideration is finite. This observation goes back to Fishburn (1970). However, Hansson (1976, p. 89) points out (quoting correspondence with Peter Fishburn) that Julian Blau was aware of the existence of non-dictatorial social welfare functions in the infinite-population case as early as 1960 without publishing this observation. Sen (1979) and Suzumura (2000) highlight the role played by the finiteness assumption in their respective methods of proving Arrow's theorem. Kirman and Sondermann (1972) and Hansson (1976) cast a new light on the structure of an Arrovian social welfare function with an infinite population, showing that the set of decisive coalitions for a social welfare function that satisfies Arrow's axioms of unlimited domain, weak Pareto and independence of irrelevant alternatives forms an ultrafilter. Their analyses apply to any (finite or infinite) population without any further structural assumptions. In an important contribution, Ferejohn and Page (1978) endowed the infinite population social choice model by adding an intertemporal component. Time flows only unidirectionally, and any two distinct members of the society (or generations) are such that one generation appears in the society after the other. As a result of introducing this time structure of infinite population, Ferejohn and Page (1978) provide a new link between Arrow's multiprofile approach to social choice and the theory of evaluating infinite intergenerational utility streams as initiated by Koopmans (1960) and Diamond (1965). In the KoopmansDiamond framework, the focus is on resource allocations among different generations with a fixed utility function for each generation. Thus, multi-profile considerations do not arise in this traditional setting.

Starting out with Hansson's (1976) result on the ultrafilter structure of the set of decisive coalitions, Ferejohn and Page (1978) propose a classical stationarity condition in an infinite-horizon multi-profile social choice model and show that if a social welfare function that satisfies their stationarity property in addition to Arrow's conditions exists, then generation one must be a dictator. Stationarity as defined by Ferejohn and Page demands that if a common first-period alternative is eliminated from two infinite streams of per-period alternatives, then the resulting continuation streams must be ranked in the same way as the original streams according to the social ranking obtained for the original profile. The reason why generation one is the only candidate for a dictator is the conjunction of the unidirectional nature of the flow of time and the resulting bias in favor of the first generation embodied in the stationarity property. Dictatorships of later 
generations cannot be stationary because we can only move forward but not backward in time.

As Ferejohn and Page (1978) note themselves, the question whether such a social welfare function exists is left open by their analysis; they show that, conditional on its existence, a stationary social welfare function satisfying Arrow's (1951; 1963; 2012) axioms must be dictatorial with generation one being the dictator. Packel (1980) resolved the existence issue by establishing a strong impossibility result: no collective choice rule that generates complete social rankings can satisfy unlimited domain, weak Pareto and stationarity. Neither transitivity of the social preference relations nor independence of irrelevant alternatives are needed for this result. Bossert and Suzumura (2011) prove a slightly stronger version of Packel's (1980) impossibility theorem by dropping completeness of the social relations from the list of requirements. It is possible to obtain further generalizations of this impossibility result. As is clear from its proof (which is provided later in the paper), only a single preference profile is required and, as a consequence, any domain that includes such a profile will produce the impossibility. For instance, Bossert and Suzumura (2011) point out that the same conclusion holds if individual preferences are restricted to those that are history-independent.

In the face of this rather strong impossibility result, a question arises naturally: what modifications to the domain assumption or to the classical stationarity condition allow us to obtain possibility results? Packel (1980) and Bossert and Suzumura (2011) choose two different paths in order to resolve the impossibility; see also Bossert and Suzumura (2010, Chapter 10) for a discussion.

Packel's (1980) approach consists of restricting the domain of a social welfare function to profiles where the individual preferences (or generation one's preferences) are themselves stationary. This domain assumption, which is plausible if social preferences are required to be stationary in Ferejohn and Page's (1978) sense, allows for the existence of social welfare functions that satisfy the remaining axioms.

Bossert and Suzumura (2011), on the other hand, consider an alternative domain assumption - namely, the assumption that each generation's preference relation is selfish in the sense that it depends on the per-period outcome for this generation only. This selfish domain also allows for the existence of social welfare functions that satisfy weak Pareto and classical stationarity. However, requiring independence of irrelevant alternatives or Pareto indifference in addition again generates impossibilities. The impossibility result involving independence does, as far as we are aware, not appear in the earlier literature. In order to circumvent these new impossibilities, the selfish domain assumption is supple- 
mented by a modification of the stationarity axiom. Especially in the context of selfish preferences, it seems natural to consider a suitable multi-profile version of stationarity. Multi-profile stationarity requires that, for any two streams of per-period alternatives and for any preference profile, if the first-period alternatives are the same in the two streams, then the social ranking of the two streams according to this profile is the same as the social ranking that results if the common first-period alternative is removed along with the preference ordering of generation one. When combined, multi-profile stationarity and selfish domain allow for social welfare functions that also satisfy weak Pareto, independence of irrelevant alternatives and Pareto indifference. Moreover, these properties can be used to characterize the lexicographic dictatorship in which the generations are taken into consideration in chronological order.

Both approaches - employing the classical stationary domain or the selfish domainallow for possibilities. However, the existence of a dictator (generation one) is implied under either of the two domain assumptions. Thus, although the infinite-population version of Arrow's social choice problem permits, in principle, non-dictatorial rules, these additional possibilities vanish in an intergenerational setting if the above-described notions of stationarity are imposed.

In this paper, we provide a brief survey of multi-profile intergenerational social choice as outlined above. After introducing the basic definitions, we provide a statement of Hansson's (1976) ultrafilter theorem which is used in several of the subsequent results. This is followed by a discussion of the fundamental Ferejohn-Page (1978) theorem and the impossibility established by Packel (1980) and generalized by Bossert and Suzumura (2011). Then the two methods of modifying the domain or the stationarity axiom and their consequences are reviewed and further possibility, impossibility and characterization results are stated. This includes the new impossibility theorem involving selfish domains and independence of irrelevant alternatives alluded to above.

We provide full proofs whenever they are based on elementary methods. Hansson's (1976) theorem is stated without a proof and we refer the reader to the original article instead. Full proofs of results that rely on variants of the ultrafilter theorem are not given; this is the case because these variants would themselves require proofs due to the different domain assumptions employed. In these cases (to be precise, Packel's (1980) possibility result and Bossert and Suzumura's (2011) characterization), however, the proof ideas are outlined in some detail to explain the intuition underlying the respective result. 


\section{Intergenerational social choice and decisiveness}

Suppose there is a set of per-period alternatives $X$ with $|X| \geq 3$. Let $X^{\infty}$ be the set of all infinite streams of per-period alternatives $\mathbf{x}=\left(x_{1}, x_{2}, \ldots\right)$ where, for each generation $t \in \mathbb{N}, x_{t} \in X$ is the period- $t$ alternative experienced by generation $t$.

The set of all binary relations on $X^{\infty}$ is denoted by $\mathcal{B}$. An ordering is a reflexive, complete and transitive relation and the set of all orderings on $X^{\infty}$ is denoted by $\mathcal{R}$. The asymmetric part and the symmetric part of a relation $R$ are denoted by $P(R)$ and $I(R)$, respectively. Furthermore, for all $\mathbf{x}, \mathbf{y} \in X^{\infty}$ and for all $R \in \mathcal{B},\left.R\right|_{\{\mathbf{x}, \mathbf{y}\}}$ is the restriction of $R$ to the set $\{\mathbf{x}, \mathbf{y}\}$.

The preference ordering of generation $t \in \mathbb{N}$ is $R_{t} \in \mathcal{R}$. A (preference) profile is a stream $\mathbf{R}=\left(R_{1}, R_{2}, \ldots\right)$ of orderings on $X^{\infty}$. The set of all such profiles is denoted by $\mathcal{R}^{\infty}$. Throughout the paper, we assume that individual preferences are orderings.

In the infinite-horizon context studied in this paper, a collective choice rule is a mapping $f: \mathcal{D} \rightarrow \mathcal{B}$, where $\mathcal{D} \subseteq \mathcal{R}^{\infty}$ with $\mathcal{D} \neq \emptyset$ is the domain of $f$. The interpretation is that, for a profile $\mathbf{R} \in \mathcal{D}, f(\mathbf{R})$ is the social ranking of streams in $X^{\infty}$. If $f(\mathbf{R})$ is an ordering for all $\mathbf{R} \in \mathcal{D}, f$ is a social welfare function.

Arrow's $(1951 ; 1963 ; 2012)$ fundamental properties are an unlimited domain assumption, the weak Pareto principle and independence of irrelevant alternatives. These axioms are well-established and require no further discussion.

Unlimited domain. $\mathcal{D}=\mathcal{R}^{\infty}$.

Weak Pareto. For all $\mathbf{x}, \mathbf{y} \in X^{\infty}$ and for all $\mathbf{R} \in \mathcal{D}$,

$$
\mathbf{x} P\left(R_{t}\right) \mathbf{y} \text { for all } t \in \mathbb{N} \Rightarrow \mathbf{x} P(f(\mathbf{R})) \mathbf{y} .
$$

Independence of irrelevant alternatives. For all $\mathbf{x}, \mathbf{y} \in X^{\infty}$ and for all $\mathbf{R}, \mathbf{R}^{\prime} \in \mathcal{D}$,

$$
\left.R_{t}\right|_{\{\mathbf{x}, \mathbf{y}\}}=\left.R_{t}^{\prime}\right|_{\{\mathbf{x}, \mathbf{y}\}} \text { for all }\left.t \in \mathbb{N} \Rightarrow f(\mathbf{R})\right|_{\{\mathbf{x}, \mathbf{y}\}}=\left.f\left(\mathbf{R}^{\prime}\right)\right|_{\{\mathbf{x}, \mathbf{y}\}} .
$$

A set $T \subseteq \mathbb{N}$ is decisive for a social welfare function $f$ if and only if, for all $\mathbf{x}, \mathbf{y} \in X^{\infty}$ and for all $\mathbf{R} \in \mathcal{D}$,

$$
\mathbf{x} P\left(R_{t}\right) \mathbf{y} \text { for all } t \in T \Rightarrow \mathbf{x} P(f(\mathbf{R})) \mathbf{y} .
$$

Clearly, $\mathbb{N}$ is decisive for any social welfare function $f$ that satisfies weak Pareto. If there is a generation $t \in \mathbb{N}$ such that $\{t\}$ is decisive for $f$, generation $t$ is a dictator for $f$ and the social welfare function $f$ is said to be dictatorial.

A filter on $\mathbb{N}$ is a collection $\mathcal{F}$ of subsets of $\mathbb{N}$ such that 
1. $\emptyset \notin \mathcal{F}$;

2. $N \in \mathcal{F}$;

3. for all $T, T^{\prime} \in \mathcal{F}, T \cap T^{\prime} \in \mathcal{F}$;

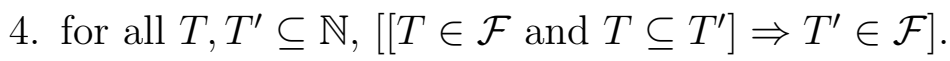

An ultrafilter on $\mathbb{N}$ is a collection $\mathcal{U}$ of subsets of $\mathbb{N}$ such that

1. $\emptyset \notin \mathcal{U}$;

2. for all $T \subseteq \mathbb{N},[T \in \mathcal{U}$ or $\mathbb{N} \backslash T \in \mathcal{U}]$;

3. for all $T, T^{\prime} \in \mathcal{U}, T \cap T^{\prime} \in \mathcal{U}$.

The conjunction of properties 1 and 2 in the definition of an ultrafilter implies that $\mathbb{N} \in \mathcal{U}$ and, furthermore, the conjunction of properties 1 and 3 implies that the disjunction in property 2 is exclusive - that is, $T$ and $\mathbb{N} \backslash T$ cannot both be in $\mathcal{U}$.

An ultrafilter $\mathcal{U}$ is principal if and only if there exists a $t \in \mathbb{N}$ such that, for all $T \subseteq \mathbb{N}$, $T \in \mathcal{U}$ if and only if $t \in T$. Otherwise, $\mathcal{U}$ is a free ultrafilter. It can be verified easily that if $\mathbb{N}$ is replaced with a finite set, then the only ultrafilters are principal and, therefore, Hansson's theorem reformulated for finite populations reduces to Arrow's (1951; 1963; 2012) theorem - that is, there exists an individual (or a generation) $t$ which is a dictator. In the infinite-population case, a set of decisive coalitions that is a principal ultrafilter corresponds to a dictatorship just as in the finite case. Unlike in the finite case, there also exist free ultrafilters but they cannot be defined explicitly; the proof of their existence relies on non-constructive methods in the sense of using variants of the axiom of choice. These free ultrafilters are non-dictatorial. However, social preferences associated with sets of decisive coalitions that form free ultrafilters fail to be continuous with respect to most standard topologies; see, for instance, Campbell (1990; 1992a,b).

Hansson (1976) shows that if a social welfare function $f$ satisfies unlimited domain, weak Pareto and independence of irrelevant alternatives, then the set of all decisive coalitions for $f$ must be an ultrafilter. For future reference, we provide a statement of Hansson's (1976) theorem formulated in the intertemporal context and refer the reader to the original paper for the proof of the more general result that applies to any population with at least two members.

Theorem 1 (Hansson, 1976) If a social welfare function $f$ satisfies unlimited domain, weak Pareto and independence of irrelevant alternatives, then the set of all decisive coalitions for $f$ is an ultrafilter. 
Hansson (1976) also shows that, for any ultrafilter $\mathcal{U}$, there exists a social welfare function $f$ that satisfies unlimited domain, weak Pareto and independence of irrelevant alternatives such that the set of decisive coalitions for $f$ is equal to $\mathcal{U}$. Moreover, he provides a parallel analysis for situations where the transitivity requirement on social rankings is weakened to quasi-transitivity. In this case, the resulting sets of decisive coalitions are filters rather than ultrafilters; see Hansson (1976) for details.

\section{Classical stationarity}

Arrow's $(1951 ; 1963 ; 2012)$ axioms introduced in the previous section are well-known from the relevant literature and require no further discussion. None of them, however, invoke the intertemporal structure of our model. Classical stationarity introduced by Ferejohn and Page (1978), in contrast, is based on the unidirectional nature of time. The underlying idea is due to Koopmans (1960) in a related but distinct context: if two streams of perperiod alternatives agree in the first period, their relative social ranking is the same as that of their respective sub-sequences from period two onward. To formulate a property of this nature in a multi-profile setting, the profile under consideration for each of the two comparisons must be specified.

First, we introduce the definition of a stationary binary relation on $X^{\infty}$. Let $t \in \mathbb{N}$. For $\mathbf{x} \in X^{\infty}$, the period- $t$ continuation of $\mathbf{x}$ is

$$
\mathbf{x}_{\geq t}=\left(x_{t}, x_{t+1}, \ldots\right),
$$

that is, $\left(\mathbf{x}_{\geq t}\right)_{\tau}=x_{\tau+t-1}$ for all $\tau \in \mathbb{N}$. Analogously, for $\mathbf{R} \in \mathcal{R}^{\infty}$, the period- $t$ continuation of $\mathbf{R}$ is

$$
\mathbf{R}_{\geq t}=\left(R_{t}, R_{t+1}, \ldots\right) .
$$

A relation $R$ on $X^{\infty}$ is stationary if and only if, for all $\mathbf{x}, \mathbf{y} \in X^{\infty}$, if $x_{1}=y_{1}$, then

$$
\mathbf{x} R \mathbf{y} \Leftrightarrow \mathbf{x}_{\geq 2} R \mathbf{y}_{\geq 2}
$$

In Ferejohn and Page's (1978) and Packel's (1980) definitions of stationarity, the same profile is employed before and after the common first-period alternative is removed. This leads to the following axiom.

Classical stationarity. For all $\mathbf{R} \in \mathcal{D}, f(\mathbf{R})$ is stationary.

Ferejohn and Page's (1978) fundamental result establishes that if there exists a social welfare function $f$ that satisfies unlimited domain, weak Pareto, independence of irrelevant 
alternatives and classical stationarity, then generation one must be a dictator for $f$. As they clearly acknowledge, the existence issue itself remains unresolved by their theorem; however, this question of existence has been resolved in the meantime (see Packel (1980) and Bossert and Suzumura (2011)). To be very clear from the outset, let us state that there exists no collective choice rule (and, thus, no social welfare function) that satisfies unlimited domain, weak Pareto and classical stationarity; see Theorem 3 below. Thus, considerably more is known now about the issue at hand than at the time when Ferejohn and Page wrote their path-breaking paper. Nevertheless, the major purpose of the present contribution is to provide a survey of the relevant literature and, since the Ferejohn-Page theorem plays such a fundamental role, we consider it imperative to present their result in detail, in spite of the observation that stronger results are available nowadays. In our opinion, a proper account of the developments in this area would be incomplete without giving the seminal contribution of Ferejohn and Page (1978) the credit and the appreciation that it deserves.

Theorem 2 (Ferejohn and Page, 1978) If a social welfare function $f$ satisfies unlimited domain, weak Pareto, independence of irrelevant alternatives and classical stationarity, then generation one is a dictator for $f$.

Proof. The proof proceeds by showing that, given the axioms in the theorem statement, $\{1\}$ is decisive and, thus, generation one is a dictator for $f$.

By Theorem 1, the set of decisive coalitions is an ultrafilter. Let $x, y \in X$ and let $\succeq$ be an ordering on $X$ such that

$$
x P(\succeq) y .
$$

Define the profile $\mathbf{R}$ as follows. Let, for all $\mathbf{x}, \mathbf{y} \in X^{\infty}$ and for all $t \in \mathbb{N}$,

$$
\mathbf{x} R_{t} \mathbf{y} \Leftrightarrow x_{t} \succeq y_{t}
$$

Now consider the streams

$$
\begin{aligned}
\mathbf{x} & =(x, x, y, x, y, x, \ldots) \\
\mathbf{y} & =(y, y, x, y, x, y, \ldots) \\
\mathbf{z} & =(x, y, x, y, x, y, \ldots)=\mathbf{x}_{\geq 2}=\mathbf{w}_{\geq 2} \\
\mathbf{w} & =(y, x, y, x, y, x, \ldots)=\mathbf{z}_{\geq 2}=\mathbf{y}_{\geq 2} .
\end{aligned}
$$

We have $\mathbf{w} P\left(R_{t}\right) \mathbf{z}$ for all even $t \in \mathbb{N}$ and $\mathbf{z} P\left(R_{t}\right) \mathbf{w}$ for all odd $t \in \mathbb{N}$. By definition of an ultrafilter, either

$$
\{t \in \mathbb{N} \mid t \text { is even }\} \text { is decisive }
$$


or

$$
\{t \in \mathbb{N} \mid t \text { is odd }\} \text { is decisive. }
$$

If (1) is true, it follows that

$$
\mathbf{w} P(f(\mathbf{R})) \mathbf{z}
$$

Because

$$
\mathbf{w}=\mathbf{z}_{\geq 2} \text { and } \mathbf{z}=\mathbf{x}_{\geq 2} \text { and }\left(\mathbf{z}_{\geq 2}\right)_{1}=\left(\mathbf{x}_{\geq 2}\right)_{1}=x
$$

classical stationarity implies

$$
\mathbf{z} P(f(\mathbf{R})) \mathbf{x}
$$

Analogously, because

$$
\mathbf{w}=\mathbf{y}_{\geq 2} \text { and } \quad \mathbf{z}=\mathbf{w}_{\geq 2} \text { and }\left(\mathbf{y}_{\geq 2}\right)_{1}=\left(\mathbf{w}_{\geq 2}\right)_{1}=y
$$

classical stationarity implies

$$
\mathbf{y} P(f(\mathbf{R})) \mathbf{w} .
$$

Using (5), (3) and (4), transitivity implies $\mathbf{y} P(f(\mathbf{R})) \mathbf{x}$. But $\mathbf{x} P\left(R_{t}\right) \mathbf{y}$ for all even $t \in \mathbb{N}$ and, thus, we obtain a contradiction to the decisiveness of $\{t \in \mathbb{N} \mid t$ is even $\}$. Therefore, (1) is false and (2) must apply.

By (2),

$$
\mathbf{z} P(f(\mathbf{R})) \mathbf{w}
$$

Because

$$
\mathbf{z}=\mathbf{x}_{\geq 2} \text { and } \mathbf{w}=\mathbf{z}_{\geq 2} \text { and }\left(\mathbf{x}_{\geq 2}\right)_{1}=\left(\mathbf{z}_{\geq 2}\right)_{1}=x
$$

classical stationarity implies

$$
\mathbf{x} P(f(\mathbf{R})) \mathbf{z}
$$

Analogously, because

$$
\mathbf{z}=\mathbf{w}_{\geq 2} \text { and } \mathbf{w}=\mathbf{y}_{\geq 2} \text { and }\left(\mathbf{w}_{\geq 2}\right)_{1}=\left(\mathbf{y}_{\geq 2}\right)_{1}=y
$$

classical stationarity implies

$$
\mathbf{w} P(f(\mathbf{R})) \mathbf{y} \text {. }
$$

Using (7), (6) and (8), transitivity implies $\mathbf{x} P(f(\mathbf{R})) \mathbf{y}$. We have

$$
\left\{t \in \mathbb{N} \mid \mathbf{x} P\left(R_{t}\right) \mathbf{y}\right\}=\{1\} \cup\{t \in \mathbb{N} \mid t \text { is even }\}
$$

and, thus, the complement of this set cannot be decisive. Therefore,

$$
\{1\} \cup\{t \in \mathbb{N} \mid t \text { is even }\}
$$


is decisive and, by property 3 of an ultrafilter, it follows that

$$
\{1\}=\{t \in \mathbb{N} \mid t \text { is odd }\} \cap(\{1\} \cup\{t \in \mathbb{N} \mid t \text { is even }\})
$$

is decisive, and the proof is complete.

Packel (1980) answers the existence question left open by Ferejohn and Page (1978) in the negative by showing that there does not exist any collective choice rule that generates complete social rankings and satisfies unlimited domain, weak Pareto and classical stationarity. Bossert and Suzumura (2011) slightly strengthen Packel's (1980) impossibility result by dropping the completeness assumption.

Theorem 3 (Packel, 1980; Bossert and Suzumura, 2011) There exists no collective choice rule $f$ that satisfies unlimited domain, weak Pareto and classical stationarity.

Proof. Suppose $f$ is a collective choice rule that satisfies the axioms of the theorem statement. Let $x, y \in X$.

For each odd $t \in \mathbb{N}$, let $\succeq_{t}$ be an antisymmetric ordering on $X$ such that

$$
y P\left(\succeq_{t}\right) x
$$

For each even $t \in \mathbb{N}$, let $\succeq_{t}$ be an antisymmetric ordering on $X$ such that

$$
x P\left(\succeq_{t}\right) y .
$$

Define a profile $\mathbf{R}$ as follows. For all $\mathbf{x}, \mathbf{y} \in X^{\infty}$, let

$$
\mathbf{x} P\left(R_{1}\right) \mathbf{y} \Leftrightarrow x_{1} P\left(\succeq_{1}\right) y_{1} \text { or }\left[x_{1}=y_{1} \text { and } x_{3} P\left(\succeq_{1}\right) y_{3}\right]
$$

Now let, for all $\mathbf{x}, \mathbf{y} \in X^{\infty}$,

$$
\mathbf{x} R_{1} \mathbf{y} \Leftrightarrow \neg \mathbf{y} P\left(R_{1}\right) \mathbf{x} .
$$

For all $t \in \mathbb{N} \backslash\{1\}$ and for all $\mathbf{x}, \mathbf{y} \in X^{\infty}$, let

$$
\mathbf{x} R_{t} \mathbf{y} \Leftrightarrow x_{t} \succeq_{t} y_{t} .
$$

Now consider the streams

$$
\begin{aligned}
& \mathbf{x}=(x, x, y, x, y, x, \ldots) \\
& \mathbf{y}=(x, y, x, y, x, y, \ldots)=\mathbf{x}_{\geq 2} \\
& \mathbf{z}=(y, x, y, x, y, x, \ldots)=\mathbf{y}_{\geq 2}
\end{aligned}
$$


We have $\mathbf{x} P\left(R_{t}\right) \mathbf{y}$ for all $t \in \mathbb{N}$ and, by weak Pareto, $\mathbf{x} P(f(\mathbf{R})) \mathbf{y}$. Stationarity implies $\mathbf{y} P(f(\mathbf{R})) \mathbf{z}$. But $\mathbf{z} P\left(R_{t}\right) \mathbf{y}$ for all $t \in \mathbb{N}$, and we obtain a contradiction to weak Pareto.

The result established in the above theorem can be strengthened by restricting the domain. Note that only a single profile is used in its proof and, thus, the impossibility remains valid if the domain is restricted to any subset containing this specific profile. For instance, Bossert and Suzumura (2011) phrase the result by using a forward-looking domain such that each generation $t$ compares any two streams exclusively on the basis of their period- $t$ continuations.

Based on the observations of this section, there appear to be two natural ways to proceed in order to arrive at possibility results.

The first of these, due to Packel (1980), is discussed in the following section. Packel (1980) retains the classical stationarity assumption throughout his analysis. To resolve the impossibility, he employs domains that only contain stationary individual preferences (or domains that only include profiles in which generation one's preference ordering must be stationary and the orderings of all other generations may be arbitrary).

The second approach involves replacing classical stationarity with a multi-profile variant of stationarity and an alternative domain restriction due to Bossert and Suzumura (2011). Multi-profile stationarity differs from classical stationarity in that not only common first-period outcomes are removed from two streams but also the preference ordering of the first generation. Bossert and Suzumura also employ selfish domains, that is, domains such that each generation's preference ordering depends on this generation's perperiod outcomes only. We illustrate the consequences of using multi-profile stationarity in a setting with selfish domains in the final section of the paper.

\section{Classical stationary domains}

The classical stationary domain $\mathcal{R}_{C}^{\infty}$ is composed of all profiles $\mathbf{R} \in \mathcal{R}^{\infty}$ such that $R_{t}$ is stationary for each $t \in \mathbb{N}$. The resulting domain assumption is used by Packel (1980).

Classical stationary domain. $\mathcal{D}=\mathcal{R}_{C}^{\infty}$.

Packel's (1980) possibility result establishes that classical stationarity is compatible with weak Pareto and independence of irrelevant alternatives on classical stationary domains even if the collective choice rule is required to produce social orderings. 
Theorem 4 (Packel, 1980) There exists a social welfare function $f$ that satisfies classical stationary domain, weak Pareto, independence of irrelevant alternatives and classical stationarity.

Proof. An example is sufficient to prove the theorem. Let, for all $\mathbf{R} \in \mathcal{R}^{\infty}, f(\mathbf{R})=R_{1}$. Because $R_{1}$ is stationary by the domain assumption, it follows immediately that all the axioms of the theorem statement are satisfied.

As is evident from Packel's (1980, p. 223) formulation of the result, the possibility survives if the domain is expanded by allowing the preference orderings of all generations other than generation one to be arbitrary; what matters is that generation one's preferences are stationary.

Although the above theorem provides a possibility result, the example invoked is not very promising - it involves a dictatorship of generation one. Indeed, this is no coincidence; as Packel (1980) shows, even if the domain is restricted so as to allow no preferences other than stationary ones for all generations, generation-one dictatorships are the only social welfare functions that satisfy weak Pareto, independence of irrelevant alternatives and classical stationarity. This observation is parallel to that of Ferejohn and Page (1978) but, in the case of the following theorem, existence is not an issue as Theorem 4 illustrates.

Theorem 5 (Packel, 1980) If a social welfare function $f$ satisfies classical stationary domain, weak Pareto, independence of irrelevant alternatives and classical stationarity, then generation one is a dictator for $f$.

Sketch of proof. The proof of this theorem is similar to that of Theorem 2. However, it is necessary to prove a variant of Hansson's (1976) result (Theorem 1) that applies to the classical stationary domain as opposed to the unlimited domain. Once this is accomplished, the remaining steps parallel those employed in the proof of Theorem 2.

\section{Selfish domains and multi-profile stationarity}

The selfish domain $\mathcal{R}_{S}^{\infty}$ is obtained by letting, for all $\mathbf{R} \in \mathcal{R}^{\infty}, \mathbf{R} \in \mathcal{R}_{S}^{\infty}$ if and only if, for each $t \in \mathbb{N}$, there exists an ordering $\succeq_{t}$ on $X$ such that, for all $\mathbf{x}, \mathbf{y} \in X^{\infty}$,

$$
\mathbf{x} R_{t} \mathbf{y} \Leftrightarrow x_{t} \succeq_{t} y_{t}
$$

Selfish domain. $\mathcal{D}=\mathcal{R}_{S}^{\infty}$. 
As an alternative to Theorem 4, we can replace unlimited domain with selfish domain instead of classical stationary domain in order to obtain a possibility result.

Theorem 6 (Bossert and Suzumura, 2011) There exists a social welfare function $f$ that satisfies selfish domain, weak Pareto and classical stationarity.

Proof. Again, an example is sufficient to prove this theorem. Suppose $\left(\succeq_{1}, \succeq_{2}, \ldots\right)$ is the profile of orderings on $X$ associated with the selfish profile $\mathbf{R} \in \mathcal{R}_{S}^{\infty}$ of orderings on $X^{\infty}$. Define a social welfare function $f$ by letting, for all $\mathbf{x}, \mathbf{y} \in X^{\infty}$ and for all $\mathbf{R} \in \mathcal{R}_{S}^{\infty}$, $\mathbf{x} f(\mathbf{R}) \mathbf{y}$ if and only if

$$
\left[x_{\tau} I\left(\succeq_{1}\right) y_{\tau} \text { for all } \tau \in \mathbb{N}\right] \text { or }
$$

[there exists $t \in \mathbb{N}$ such that $x_{\tau} I\left(\succeq_{1}\right) y_{\tau}$ for all $\tau<t$ and $x_{t} P\left(\succeq_{1}\right) y_{t}$.

That $f$ satisfies selfish domain follows immediately by definition.

To see that weak Pareto is satisfied, note first that, according to $f$ as defined above,

$$
\mathbf{x} P\left(R_{t}\right) \mathbf{y}
$$

is equivalent to

$$
x_{t} P\left(\succeq_{t}\right) y_{t}
$$

for all $t \in \mathbb{N}$. Thus, $x_{\tau} I\left(\succeq_{1}\right) y_{\tau}$ for all $\tau<1$ and $x_{1} P\left(\succeq_{1}\right) y_{1}$ (the indifference relations are vacuously true because the set of periods $\tau$ such that $\tau<1$ is empty). By definition of $f$, it follows that $\mathbf{x} P(f(\mathbf{R})) \mathbf{y}$.

It remains to establish that $f$ satisfies classical stationarity. Suppose the alternatives $\mathbf{x}, \mathbf{y} \in X^{\infty}$ and the stationary profile $\mathbf{R} \in \mathcal{R}_{S}^{\infty}$ are such that $x_{1}=y_{1}$. Thus, because $\succeq_{1}$ is an ordering (and, thus, reflexive), we obtain $x_{1} I\left(\succeq_{1}\right) y_{1}$. It follows that

$$
\begin{aligned}
\mathbf{x}_{\geq 2} f(\mathbf{R}) \mathbf{y}_{\geq 2} \Leftrightarrow & {\left[x_{\tau} I\left(\succeq_{1}\right) y_{\tau} \text { for all } \tau \in \mathbb{N} \backslash\{1\} \text { and } x_{1} I\left(\succeq_{1}\right) y_{1}\right] \text { or } } \\
& {\left[\text { there exists } t \in \mathbb{N} \backslash\{1\} \text { such that } x_{\tau} I\left(\succeq_{1}\right) y_{\tau} \text { for all } \tau<t\right.} \\
& \text { and } \left.x_{t} P\left(\succeq_{1}\right) y_{t}\right] \\
\Leftrightarrow \quad & {\left[x_{\tau} I\left(\succeq_{1}\right) y_{\tau} \text { for all } \tau \in \mathbb{N}\right] \text { or } } \\
& {\left[\text { there exists } t \in \mathbb{N} \text { such that } x_{\tau} I\left(\succeq_{1}\right) y_{\tau} \text { for all } \tau<t \text { and } x_{t} P\left(\succeq_{1}\right) y_{t}\right] } \\
\Leftrightarrow & \mathbf{x} f(\mathbf{R}) \mathbf{y} .
\end{aligned}
$$

Unlike Theorem 4, the statement of Theorem 6 does not include independence of irrelevant alternatives as one of the axioms that can be satisfied under the alternative domain 
assumption. In fact, adding the independence property to the list of axioms leads to another impossibility. This is a new observation that, to the best of our knowledge, does not appear in the previous literature.

Theorem 7 There exists no collective choice rule $f$ that satisfies selfish domain, weak Pareto, independence of irrelevant alternatives and classical stationarity.

Proof. Suppose $f$ is a collective choice rule that satisfies the axioms of the theorem statement. Let $x, y, z \in X$.

For each $t \in \mathbb{N}$, let $\succeq_{t}$ be an ordering on $X$ such that

$$
y P\left(\succeq_{t}\right) x \text { and } x P\left(\succeq_{t}\right) z
$$

Furthermore, for each odd $t \in \mathbb{N}$, let $\succeq_{t}^{\prime}$ be an ordering on $X$ such that

$$
y P\left(\succeq_{t}^{\prime}\right) z \text { and } z P\left(\succeq_{t}^{\prime}\right) x
$$

Finally, for each even $t \in \mathbb{N}$, let $\succeq_{t}^{\prime}$ be an ordering on $X$ such that

$$
x P\left(\succeq_{t}^{\prime}\right) y \text { and } y P\left(\succeq_{t}^{\prime}\right) z
$$

Define two profiles $\mathbf{R}$ and $\mathbf{R}^{\prime}$ as follows. For all $\mathbf{x}, \mathbf{y} \in X^{\infty}$ and for all $t \in \mathbb{N}$, let

$$
\mathbf{x} R_{t} \mathbf{y} \Leftrightarrow x_{t} \succeq_{t} y_{t}
$$

and

$$
\mathbf{x} R_{t}^{\prime} \mathbf{y} \Leftrightarrow x_{t} \succeq_{t}^{\prime} y_{t}
$$

Clearly, the profiles thus defined are in $\mathcal{R}_{S}^{\infty}$.

Now consider the streams

$$
\begin{aligned}
\mathbf{x} & =(z, x, y, x, y, x, y, \ldots) \\
\mathbf{y} & =(z, z, x, z, x, z, x, \ldots) \\
\mathbf{z} & =(x, y, x, y, x, y, x, \ldots)=\mathbf{x}_{\geq 2} \\
\mathbf{w} & =(z, x, y, x, y, x, y, \ldots)=\mathbf{y}_{\geq 2}
\end{aligned}
$$

We have $\mathbf{z} P\left(R_{t}\right) \mathbf{w}$ for all $t \in \mathbb{N}$ and, by weak Pareto, $\mathbf{z} P(f(\mathbf{R})) \mathbf{w}$. Stationarity implies

$$
\mathbf{x} P(f(\mathbf{R})) \mathbf{y}
$$


Furthermore, w $P\left(R_{t}^{\prime}\right) \mathbf{z}$ for all $t \in \mathbb{N}$ and, using weak Pareto again, w $P\left(f\left(\mathbf{R}^{\prime}\right)\right) \mathbf{z}$. Stationarity implies

$$
\mathbf{y} P\left(f\left(\mathbf{R}^{\prime}\right)\right) \mathbf{x}
$$

But we also have

$$
\mathbf{x} I_{1} \mathbf{y} \text { and } \mathbf{x} I_{1}^{\prime} \mathbf{y}
$$

and

$$
\mathbf{x} P_{t} \mathbf{y} \text { and } \mathbf{x} P_{t}^{\prime} \mathbf{y}
$$

for all $t \in \mathbb{N} \backslash\{1\}$ which, by independence of irrelevant alternatives, requires that

$$
\mathbf{x} f(\mathbf{R}) \mathbf{y} \Leftrightarrow \mathbf{x} f\left(\mathbf{R}^{\prime}\right) \mathbf{y}
$$

contradicting the conjunction of (9) and (10).

Yet another impossibility emerges if Pareto indifference is used instead of independence of irrelevant alternatives. Pareto indifference is the analogue of weak Pareto that is obtained by replacing each occurrence of a strict preference with an indifference.

Pareto indifference. For all $\mathbf{x}, \mathbf{y} \in X^{\infty}$ and for all $\mathbf{R} \in \mathcal{D}$,

$$
\mathbf{x} I\left(R_{t}\right) \mathbf{y} \text { for all } t \in \mathbb{N} \Rightarrow \mathbf{x} I(f(\mathbf{R})) \mathbf{y}
$$

Replacing independence of irrelevant alternatives with Pareto indifference leaves the incompatibility stated in the previous theorem intact, as shown by Bossert and Suzumura (2011).

Theorem 8 (Bossert and Suzumura, 2011) There exists no collective choice rule $f$ that satisfies selfish domain, weak Pareto, Pareto indifference and classical stationarity.

Proof. Suppose $f$ is a collective choice rule that satisfies the axioms of the theorem statement. Let $x, y, z \in X$.

For each odd $t \in \mathbb{N}$, let $\succeq_{t}$ be an ordering on $X$ such that

$$
z P\left(\succeq_{t}\right) x \text { and } x I\left(\succeq_{t}\right) y
$$

For each even $t \in \mathbb{N}$, let $\succeq_{t}$ be an ordering on $X$ such that

$$
z I\left(\succeq_{t}\right) x \text { and } x P\left(\succeq_{t}\right) y .
$$


Define a profile $\mathbf{R}$ as follows. For all $\mathbf{x}, \mathbf{y} \in X^{\infty}$ and for all $t \in \mathbb{N}$, let

$$
\mathbf{x} R_{t} \mathbf{y} \Leftrightarrow x_{t} \succeq_{t} y_{t}
$$

Clearly, the profile thus defined is in $\mathcal{R}_{S}^{\infty}$.

Now consider the streams

$$
\begin{aligned}
\mathbf{x} & =(z, z, x, z, x, z, x, \ldots) \\
\mathbf{y} & =(z, x, y, x, y, x, y, \ldots) \\
\mathbf{z} & =(z, x, z, x, z, x, \ldots)=\mathbf{x}_{\geq 2} \\
\mathbf{w} & =(x, y, x, y, x, y, \ldots)=\mathbf{y}_{\geq 2} .
\end{aligned}
$$

We have $\mathbf{x} I\left(R_{t}\right) \mathbf{y}$ for all $t \in \mathbb{N}$ and, by Pareto indifference, $\mathbf{x} I(f(\mathbf{R})) \mathbf{y}$. Stationarity implies $\mathbf{z} I(f(\mathbf{R})) \mathbf{w}$. But $\mathbf{z} P\left(R_{t}\right) \mathbf{w}$ for all $t \in \mathbb{N}$, and we obtain a contradiction to weak Pareto.

The two theorems just established suggest that the selfish domain assumption can only yield satisfactory possibilities if the classical stationary assumption is amended as well. There is a plausible alternative version according to which the (common) first-period component is eliminated not only from the streams to be compared but also from the profile for which the social ranking is to be determined. The resulting axiom, which appears to be suitable in conjunction with the path chosen by focusing on selfish preferences, is due to Bossert and Suzumura (2011).

Multi-profile stationarity. For all $\mathbf{x}, \mathbf{y} \in X^{\infty}$ and for all $\mathbf{R} \in \mathcal{D}$, if $x_{1}=y_{1}$, then

$$
\mathbf{x} f(\mathbf{R}) \mathbf{y} \Leftrightarrow \mathbf{x}_{\geq 2} f\left(\mathbf{R}_{\geq 2}\right) \mathbf{y}_{\geq 2} .
$$

If multi-profile stationarity is used instead of classical stationarity, both independence of irrelevant alternatives and Pareto indifference can be accommodated in addition to selfish domain and weak Pareto. A social welfare function that satisfies multi-profile stationarity in conjunction with selfish domain, weak Pareto, independence of irrelevant alternatives and Pareto indifference is the chronological dictatorship, to be introduced shortly. In fact, the chronological dictatorship is the only social welfare function satisfying this list of axioms and, thus, it can be characterized by means of this set of properties.

The chronological dictatorship is the social welfare function $f$ defined by letting, for all $\mathbf{x}, \mathbf{y} \in X^{\infty}$ and for all $\mathbf{R} \in \mathcal{R}_{S}^{\infty}, \mathbf{x} f(\mathbf{R}) \mathbf{y}$ if and only if

$$
\left[x_{\tau} I\left(\succeq_{\tau}\right) y_{\tau} \text { for all } \tau \in \mathbb{N}\right] \text { or }
$$

[there exists $t \in \mathbb{N}$ such that $x_{\tau} I\left(\succeq_{\tau}\right) y_{\tau}$ for all $\tau<t$ and $x_{t} P\left(\succeq_{t}\right) y_{t}$ ]. 
The chronological dictatorship is, evidently, a special case of a dictatorial social welfare function and, thus, it turns out that the two alternative paths towards a resolution of Ferejohn and Page's (1978) impossibility lead to similar results. Both Packel's (1980) approach based on stationary individual preferences and Bossert and Suzumura's (2011) attempt to use selfish individual preferences in conjunction with a new version of stationarity allow for the existence of social welfare functions with the desired properties. But, due to the bias in favor of generation one that is imposed by either form of stationarity (in conjunction with the unidirectional nature of the flow of time), the resulting rules must be dictatorial with generation one being the dictator. We conclude this survey with a statement and proof sketch of Bossert and Suzumura's (2011) characterization.

Theorem 9 (Bossert and Suzumura, 2011) A social welfare function $f$ satisfies selfish domain, weak Pareto, independence of irrelevant alternatives, Pareto indifference and multi-profile stationarity if and only if $f$ is the chronological dictatorship.

Sketch of proof. That the chronological dictatorship satisfies the axioms of the theorem statement is straightforward to verify.

In order to prove the reverse implication, a version of Hansson's (1976) ultrafilter theorem (the theorem stated as Theorem 1 in Section 2 of the present paper) that applies to the selfish domain needs to be established, as is the case for Theorem 5. However, in the selfish case, Pareto indifference is required as an additional axiom. A modification of this nature is called for because the selfish domain is not sufficiently rich to generate arbitrary rankings of all streams. For example, whenever we have two streams $\mathbf{x}$ and $\mathbf{y}$ such that $x_{t}=y_{t}$ for some selfish generation $t \in \mathbb{N}$, this selfish generation must declare $\mathbf{x}$ and $\mathbf{y}$ indifferent; this is an immediate consequence of the conjunction of selfish domain and reflexivity. This addition of Pareto indifference to the list of axioms is necessitated by the observation that a fundamental preliminary result - an adaptation of Sen's (1995, p.4) field expansion lemma to our selfish domain setting - fails to be true if merely selfish domain, weak Pareto and independence of irrelevant alternatives are imposed. Loosely speaking, the field expansion lemma establishes that a decisiveness property over a given pair of alternatives can be expanded to all pairs of alternatives, thus producing full decisiveness from a weaker version that is restricted to a pair.

The proof of Theorem 9 consists of the following steps.

First, a version of the field expansion lemma for the selfish domain is proven, provided that $f$ satisfies weak Pareto, independence of irrelevant alternatives and Pareto indifference. 
In a second step, this result is used to establish a version of Hansson's (1976) ultrafilter theorem that applies to the selfish domain. Again, Pareto indifference is required in order to invoke the above-described variant of the field expansion lemma.

The third step consists of showing that the axioms imply that generation one must be a dictator for $f$. This step parallels the corresponding step in the proofs of Theorems 2 and 5, except that multi-profile stationarity is used instead of classical stationarity.

Finally, the observation that generation one is a dictator is used to show that only the chronological dictatorship satisfies the required axioms. Because the proof method employed in this step does not appear in any of the proofs outlined earlier, we provide the details.

Because $f$ is assumed to be a social welfare function (and, thus, produces social orderings for all profiles in its domain), it is sufficient to show that, for all $\mathbf{x}, \mathbf{y} \in X^{\infty}$ and for all $\mathbf{R} \in \mathcal{R}_{S}^{\infty}, \mathbf{x} P(f(\mathbf{R})) \mathbf{y}$ whenever $\mathbf{x}$ is strictly preferred to $\mathbf{y}$ according to the chronological dictatorship (the corresponding implication involving indifference is trivially satisfied because of Pareto indifference).

Suppose $t \in \mathbb{N}, \mathbf{x}, \mathbf{y} \in X^{\infty}$ and $\mathbf{R} \in \mathcal{R}_{S}^{\infty}$ are such that

$$
x_{\tau} I\left(\succeq_{\tau}\right) y_{\tau} \text { for all } \tau<t \text { and } x_{t} P\left(\succeq_{t}\right) y_{t}
$$

If $t=1$, let $\mathbf{z}=\mathbf{y}$; if $t \geq 2$, let $\mathbf{z}=\left(x_{1}, \ldots, x_{t-1}, \mathbf{y}_{\geq t}\right)$. By Pareto indifference, $\mathbf{y} I(f(\mathbf{R})) \mathbf{z}$. Transitivity implies

$$
\mathbf{x} f(\mathbf{R}) \mathbf{y} \Leftrightarrow \mathbf{x} f(\mathbf{R}) \mathbf{z}
$$

Together with the application of multi-profile stationarity $t-1$ times and noting that $\mathbf{z}_{\geq t}=\mathbf{y}_{\geq t}$, we obtain

$$
\mathbf{x} f(\mathbf{R}) \mathbf{y} \Leftrightarrow \mathbf{x} f(\mathbf{R}) \mathbf{z} \Leftrightarrow \mathbf{x}_{\geq t} f\left(\mathbf{R}_{\geq t}\right) \mathbf{z}_{\geq t} \Leftrightarrow \mathbf{x}_{\geq t} f\left(\mathbf{R}_{\geq t}\right) \mathbf{y}_{\geq t}
$$

Because generation one is a dictator for $f$ as established in the previous step, the relative ranking of $\mathbf{x}_{\geq t}$ and $\mathbf{y}_{\geq t}$ according to $\mathbf{R}_{\geq t}$ is determined by the strict preference for $\mathbf{x}$ over $\mathbf{y}$ according to the first generation in the profile $\mathbf{R}_{\geq t}$ (which is generation $t$ in $\mathbf{R}$ ), so that $\mathbf{x}_{\geq t} P\left(f\left(\mathbf{R}_{\geq t}\right)\right) \mathbf{y}_{\geq t}$ and, by $(11), \mathbf{x} P(f(\mathbf{R})) \mathbf{y}$. 


\section{References}

Arrow, K.J. (1951, second ed. 1963), Social Choice and Individual Values, Wiley, New York. Third ed., Yale University Press, New Haven, 2012.

Bossert, W. and K. Suzumura (2010), Consistency, Choice, and Rationality, Harvard University Press, Cambridge, Mass.

Bossert, W. and K. Suzumura (2011), Multi-profile intergenerational social choice, Social Choice and Welfare 37, 493-509.

Campbell, D.E. (1990), Intergenerational social choice without the Pareto principle, Journal of Economic Theory 50, 414-423.

Campbell, D.E. (1992a), Quasitransitive intergenerational social choice for economic environments, Journal of Mathematical Economics 21, 229-247.

Campbell, D.E. (1992b), Equity, Efficiency, and Social Choice, Clarendon Press, Oxford.

Diamond, P. (1965), The evaluation of infinite utility streams, Econometrica 33, 170177.

Ferejohn, J. and T. Page (1978), On the foundations of intertemporal choice, American Journal of Agricultural Economics 60, 269-275.

Fishburn, P.C. (1970), Arrow's impossibility theorem: concise proof and infinite voters, Journal of Economic Theory 2, 103-106.

Hansson, B. (1976), The existence of group preference functions, Public Choice 38, 89-98.

Kirman, A.P. and D. Sondermann (1972), Arrow's theorem, many agents, and invisible dictators, Journal of Economic Theory 5, 267-277.

Koopmans, T.C. (1960), Stationary ordinal utility and impatience, Econometrica 28, $287-309$.

Packel, E. (1980), Impossibility results in the axiomatic theory of intertemporal choice, Public Choice 35, 219-227. 
Sen, A.K. (1979), Personal utilities and public judgements: or what's wrong with welfare economics?, Economic Journal 89, 537-558. Reprinted in: A.K. Sen (1982), Choice, Welfare and Measurement, Basil Blackwell, Oxford, 327-352.

Sen, A.K. (1995), Rationality and social choice, American Economic Review 85, 1-24. Reprinted in: A.K. Sen (2002), Rationality and Freedom, The Belknap Press of Harvard University Press, Cambridge, Mass., 261-299.

Suzumura, K. (2000), Welfare economics beyond welfarist-consequentialism, Japanese Economic Review 51, 1-32. 